\title{
Diseño e Implementación de un Sistema de Videogrametría Digital para Análisis de Saltabilidad en Deportistas
}

\section{Design and Implementation of a Digital Videogrammetry System Applied to Jumping Sportsmen Analysis}

\author{
José de Jesús Salgado Patrón ${ }^{1}$ y Diego Fernando Perdomo Ortiz ${ }^{2}$
}

\begin{abstract}
Resumen
Este articulo presenta el diseño e implementación de un sistema para el análisis cinemático y cinético del salto vertical. El sistema se basa en el uso de técnicas de videogrametría y procesamiento de imágenes para registrar la secuencia del movimiento ejecutado. Se diseñó y desarrolló un programa aplicativo para el registro y procesamiento de los datos obtenidos por medio de la cámara, utilizando las herramientas de procesamiento digital de imágenes y la interface de usuario GUI que ofrece Matlab. En el programa se dispone de un modelo biomecánico bidimensional de los miembros inferiores que, a partir de cinco puntos anatómicos referenciados, determina los valores de: recorrido, velocidad, aceleración, fuerza, potencia y ángulos articulares de los miembros inferiores, a lo largo de la ejecución del salto. Se realizaron pruebas experimentales con cinco sujetos utilizando un protocolo para el salto vertical, para comparar las curvas entregadas por el software con respecto a las curvas teóricas encontradas en la literatura y datos obtenidos por otros sistemas de medición. A pesar de las limitaciones del sistema referentes al análisis bidimensional del salto vertical, se lograron obtener resultados satisfactorios con referencia a otros métodos utilizados actualmente y a instrumentos específicos para mediciones.
\end{abstract}

\section{Palabras Clave}

Videogrametría; Salto Vertical; Biomecánica; Ángulos Articulares, Procesamiento Digital de Imágenes.

\begin{abstract}
This paper presents the design and implementation of a system for kinematic and kinetic analysis of vertical jump. The system is based on the use of techniques of videogrammetry and digital image processing to register the sequence of the movement executed. An application program for the registering and processing of data obtained through the camera, using the tools of digital image processing and the user interface GUI offered by Matlab, was designed and developed. A bidimensional biomechanical model of the lower limbs is available by the program, which, from five referenced anatomical points, determines the values of distance, speed, acceleration, strength, power and joint angles of the lower limbs, along the implementation of jump. There were pilot tests carried out with five subjects using a vertical jump protocol, in order to compare the curves delivered by the software with the curves which were found theoretically in the literature and obtained data by another measurement system. In spite of the limitations of the system related to the analysis of two-dimensional vertical jump, it was possible to obtain satisfactory results with reference to other methods currently used and to specific measuring equipment.
\end{abstract}

\section{Keywords}

Videogrammetry; Vertical Jump; Biomechanics; Joint Angles, Digital Image Processing.

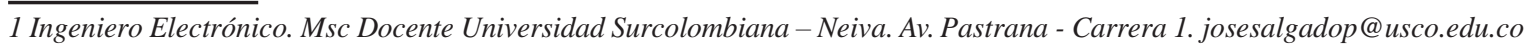

2 Ingeniero Electrónico. Universidad Surcolombiana-Neiva. Av. Pastrana - Carrera 1.diegopor_2788@hotmail.com 


\section{Introducción}

La acción de saltar es considerada como una de las cualidades básicas en un deportista que determina ciertos niveles de potencia, rapidez, coordinación, fuerza y velocidad, cuando se evalúa funcionalmente su estado físico, su proyección y su retroalimentación(Ortiz, 2004). El hecho de aplicar una fuerza músculo esquelética que genera una velocidad inicial del cuerpo en un determinado ángulo de despegue y en completa resistencia o contraria a la fuerza de gravedad para obtener una determinada distancia, establece la complejidad del mismo. Las evaluaciones de saltabilidad tienen como objetivo determinar la fuerza contráctil, potencia absoluta y relativa así como características elásticas de las extremidades inferiores (Ortiz, 2004).

El desarrollo de sistemas de videogrametría digital conforma una parte fundamental dentro del campo de la medicina deportiva o del estudio de la biomecánica (Martínez, 2009), ya que permite a los especialistas realizar el análisis tridimensional o bidimensional de variables y curvas que caracterizan la cinemática y cinética del movimiento del cuerpo humano, logrando de esta manera corregir errores en los movimientos efectuados. Se pueden ver algunas investigaciones en: (Díaz et al., 2006), (Royo, 2005) y (Rodríguez, 2005). En el campo de la medicina deportiva se hace indispensable contar con sistemas de videogrametría digital en dos dimensiones (2-D) para analizar los movimientos efectuados en el salto, que sean capaces de realizar diversos análisis de todas y cada una de las variables cinéticas y cinemáticas que influyen en la manifestación de los miembros inferiores del sujeto; algunas investigaciones nacionales e internacionales de rendimiento deportivo muestran resultados eficientes en técnicas para obtener mejores niveles de fuerza y potencia muscular: (Acevedo, 2008), (Calderón, 2004) y (García, 2004).

Actualmente en el Laboratorio de Evaluación y Desarrollo del Rendimiento Físico LEDRF ALTIUS de la Universidad Surcolombiana, las técnicas para el análisis de saltabilidad consisten en una serie de saltos sobre una plataforma, la cual es capaz de medir tiempos de vuelo y tiempos de contacto de cada salto determinando el desplazamiento del centro de gravedad de cada sujeto en cada impulso, siendo posible a través de estos datos determinar de forma indirecta las propiedades músculo-esqueléticas y diagnosticar el rendimiento de los deportistas. Por lo tanto, es una oportunidad enorme el poder incursionar en tan importante campo, donde se podría mostrar ante el mercado de la instrumentación deportiva nacional e internacional un sistema de videogrametría que obtiene de forma indirecta, variables de potencia y fuerza muscular a través del salto vertical, además de otras variables importantes generadas en la misma ejecución, evitando que las necesidades de instrumentación sean suplidas fuera del país.

\section{Metodología}

El desarrollo completo del sistema de videogrametría se describe en el diagrama de bloques que se expone en la Figura 1. Para las cuatro últimas etapas del diagrama de bloques se desarrolló una aplicación para Windows utilizando la interfaz gráfica de Matlab - Guide.

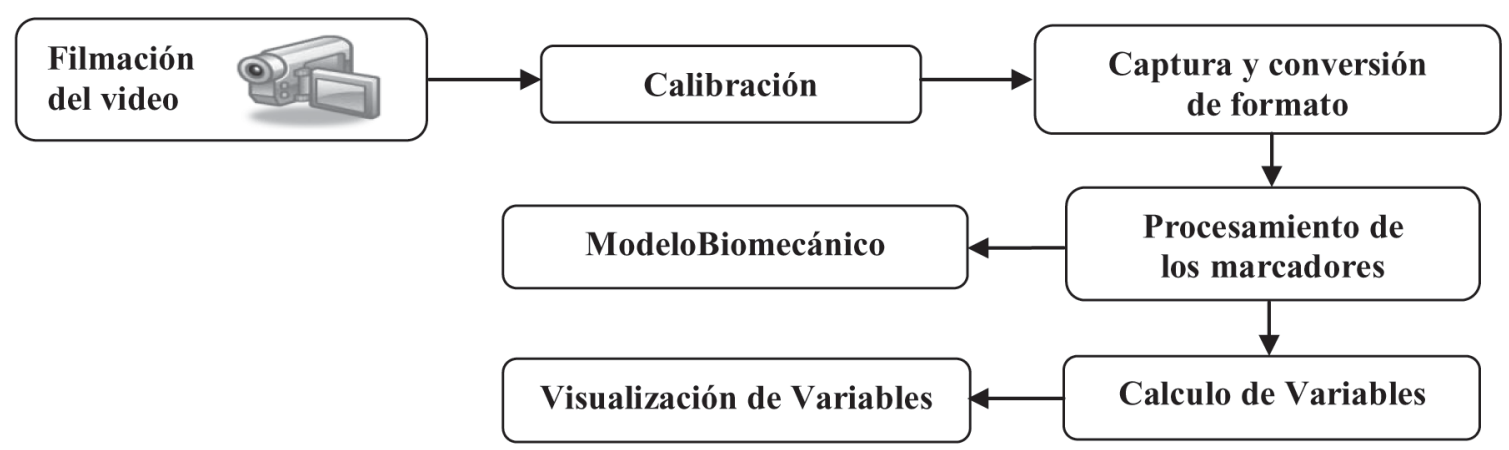

Figura 1. Diagrama de Bloques del sistema

\subsection{Filmación del Video}

Es necesario tener algunos procedimientos estandarizados para la repetitividad en las pruebas ejecutadas con los sujetos a evaluar. Por consiguiente la filmación del video está dividida en tres secciones las cuales se deben tener en cuenta siempre que se vaya a realizar una prueba de salto vertical. 
.1.1 Escenario de Filmación. El escenario de filmación debe tener las dimensiones suficientes para colocar la cámara a $2.60 \mathrm{~m}$ del plano de filmación. La distancia del centro óptico de la cámara debe estar a $80 \mathrm{~cm}$ del piso. La iluminación de la escena debe estar de forma que la luz esté de frente al plano de calibración, es decir que la iluminación esté en el mismo sentido en que la cámara esté filmando, estas dimensiones se observan en la Figura 2.

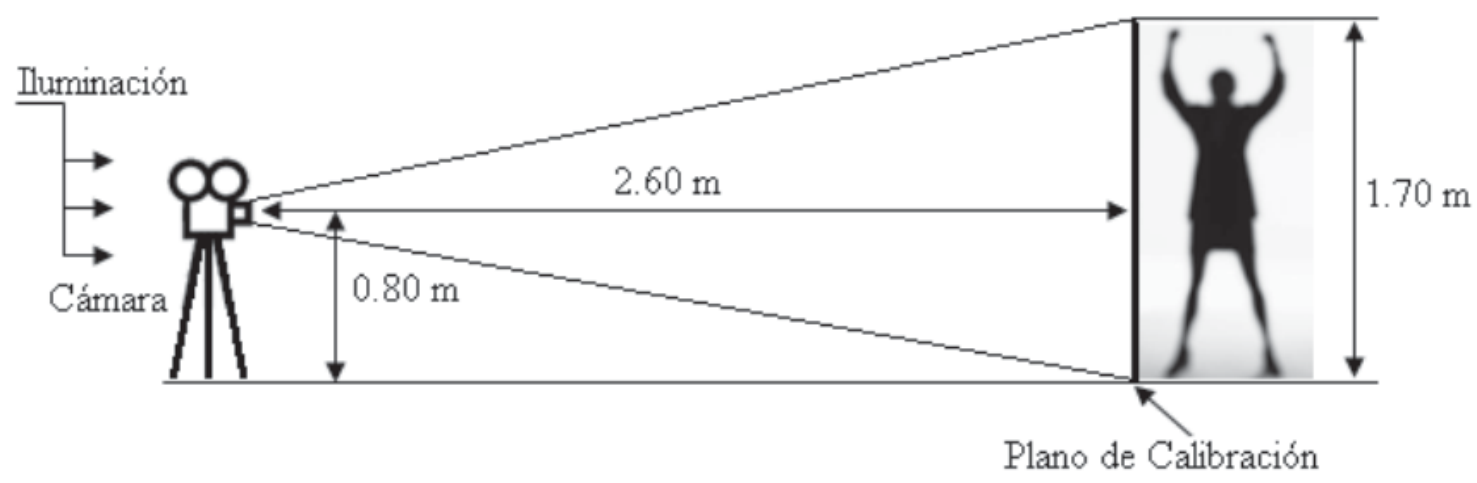

Figura 2. Dimensiones del escenario de filmación

.1.2 Ubicación de Marcadores. Se ubican cinco marcadores en el lado derecho de la extremidad inferior del deportista, independientemente del hemisferio predominante en el sujeto. En orden descendente los cinco puntos son: la aproximación del Centro de Gravedad (CG), Trocánter mayor (TM), Epicóndilo femoral lateral (EFL), Maléolo lateral (ML) y Cabeza de quinto metatarsiano (MET), tal como se ven en la Figura 3.

2.1.3 Protocolo para el Salto Vertical. Para ejecutar el salto vertical se procede primero a tomar el peso corporal del sujeto. Después que el sujeto tenga los marcadores corporales se procede a ubicarlo en el escenario de filmación, de tal forma, que estará posicionado con el plano sagital derecho del cuerpo hacia la videocámara.

\subsection{Calibración}

En la calibración se corrigen los errores de rotación y perspectiva producidos por la ubicación de la cámara, Utilizando la base mostrada en la Figura 4; con el fin de obtener una equivalencia del píxel con una medida métrica.

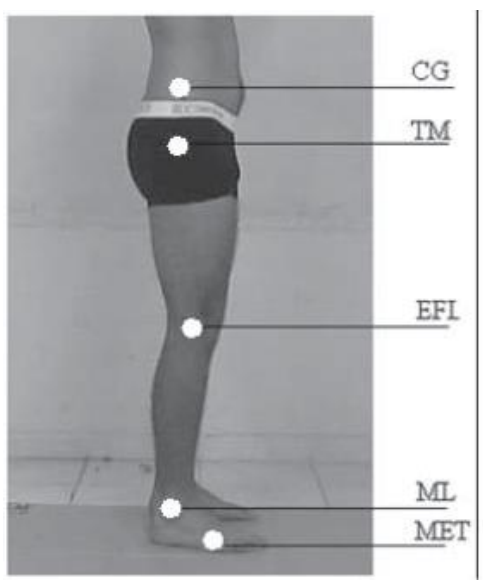

Figura 3. Ubicación de los cinco marcadores

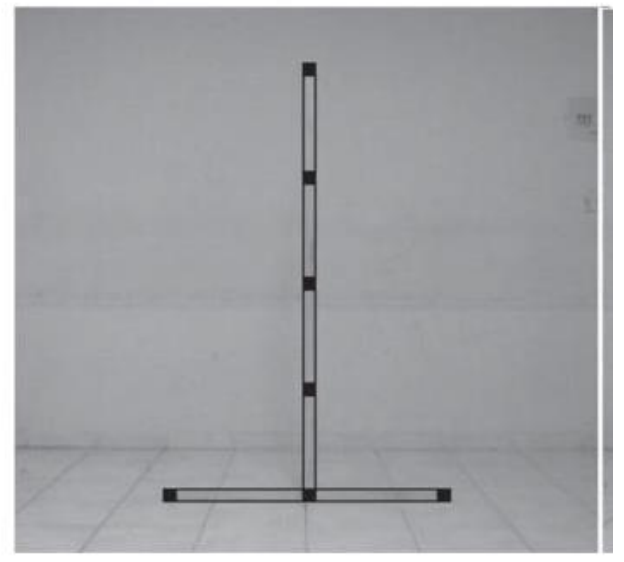

Figura 4. Base utilizada para calibración

\subsection{Captura y Conversión de Formatos}

En el proyecto se descarga el video de la cámara al computador mediante el uso de un cable USB 1.1 y se realizan las conversiones entre formatos con el conversor TMPE Gnc 3.0 XPres. Al final de esta etapa se obtiene un video en formato AVI, el cual será procesado por el software aplicativo.

\subsection{Procesamiento de los Marcadores}

El procesamiento de los marcadores se realiza con las herramientas que ofrece el Toolbox de Procesamiento de Imágenes de Matlab. 
2.4.1 Segmentación. El método utilizado para la segmentación de los marcadores fue la segmentación por color mediante la distancia Euclideana, debido a que con ésta se obtuvo un mejor rendimiento en el procesamiento.

2.4.2.Mejoramiento de la Imagen Segmentada. Se aplican operaciones morfológicas con el fin de mejorar los marcadores reconocidos sobre imágenes binarias, debido a que en algunos pixeles que forman parte del marcador se detallan espacios que no pertenecen a la binarización requerida.

2.4.3. Descripción y Extracción de Características. Se discriminan algunos objetos que cumplieron con la segmentación realizada, es así como se observan objetos que no hacen parte de los marcadores; por lo tanto se eliminan con dos discriminantes, el primero es eliminación por área y el segundo discriminante consiste en eliminar objetos que no cumplan condiciones de forma de los marcadores.

\subsection{Modelo Biomecánico}

Se muestra un modelo biomecánico de seguimiento en el salto vertical el cual está representado como una estructura virtual de dos dimensiones. Ésta representación se construye a partir de tres líneas rojas que simulan los miembros inferiores como cuerpos rígidos, estos son: el muslo, la pierna y el pie; y cinco puntos verdes, de los cuales dos determinan los centros de giro de las articulaciones: la rodilla (entre el muslo y la pierna) y el tobillo (entre la pierna y el pie).

\subsection{Cálculo de Variables}

Se calcularon a través del primer marcador corporal (CG) las variables cinemáticas, tales como el desplazamiento, velocidad y aceleración; y las cinéticas como fuerza y potencia, además de ángulos articulares obtenidos tanto en rodilla como en tobillo.

\subsection{Visualización de Gráficas}

Se presenta al usuario final una representación gráfica de fácil interpretación, que para este proyecto utilizó la interfaz gráfica de usuario GUI de Matlab. Se visualizan siete gráficas, todas con respecto al tiempo de duración del salto, estas son: Recorrido del CG, Velocidad del CG, Aceleración del CG, Fuerza Vertical, Potencia Mecánica, Ángulo articular de rodilla y Ángulo articular de tobillo.

\section{Resultados}

\subsection{Resultados del Procesamiento de los marcadores}

En la Figura 5 se observa la aplicación de operaciones morfológicas luego de obtener los cinco marcadores deseados mediante de la segmentación por color. En la imagen (a) se han encerrado tres objetos incompletos en formas, en la imagen (b) se observa la operación de dilatación, y en la imagen (c) la aplicación de la erosión.

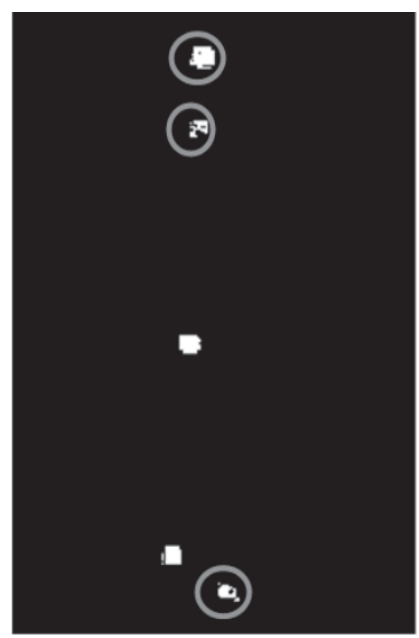

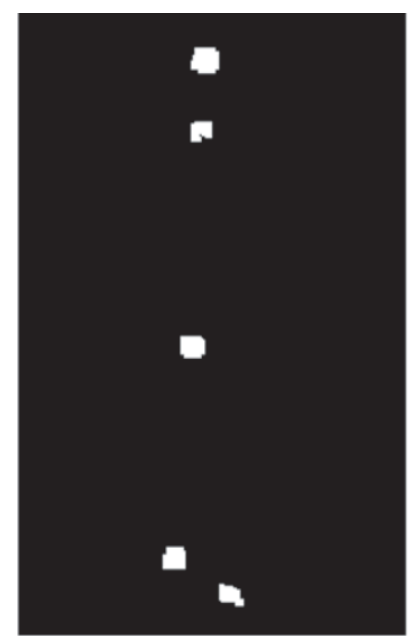

(a)

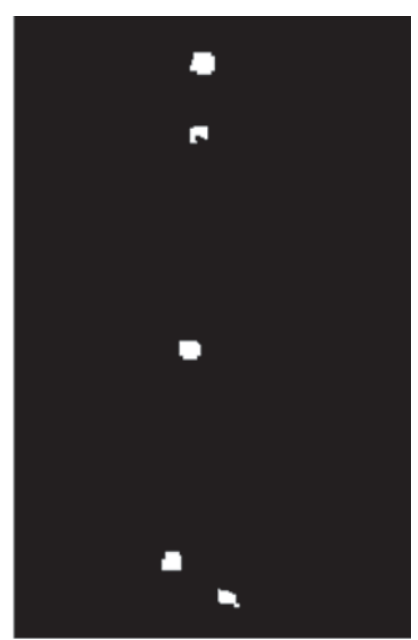

(b) (c)

Figura 5. Aplicación de operaciones morfológicas 
En la Figura 6 se observa la aplicación de los discriminantes de objetos no deseados. En la imagen (a) se observan dos falsos positivos que no cumplen en primera instancia con las condiciones de área ni de tamaño, y en la imagen (b) se muestra la imagen solamente con los objetos que son los marcadores reales.

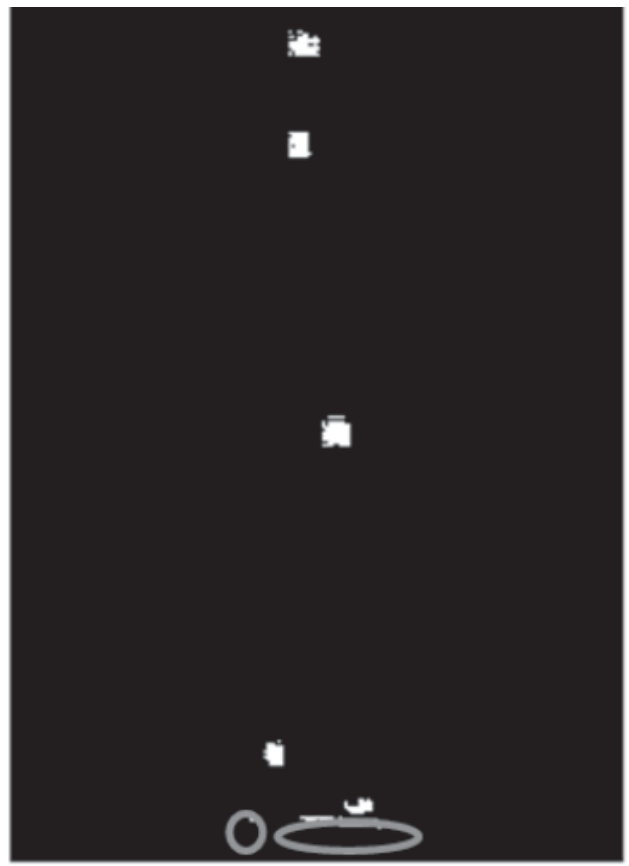

(a)

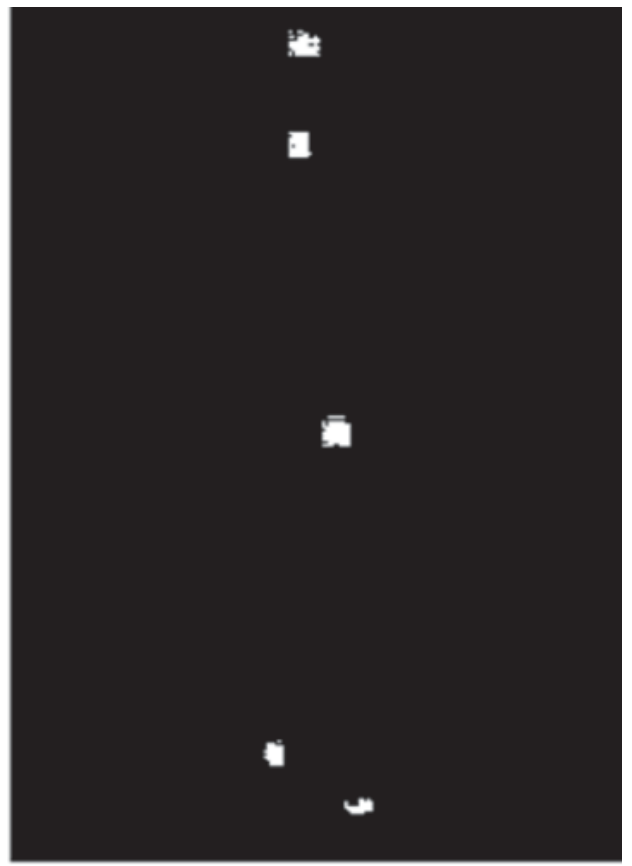

(b)

Figura 6. Aplicación de discriminantes en objetos no deseados

\subsection{Panel Central y Ejecución del Software}

$\mathrm{Al}$ abrir el software aplicativo se encuentra el panel central mostrado en la Figura 7, éste contiene cinco paneles importantes para su ejecución y visualización.

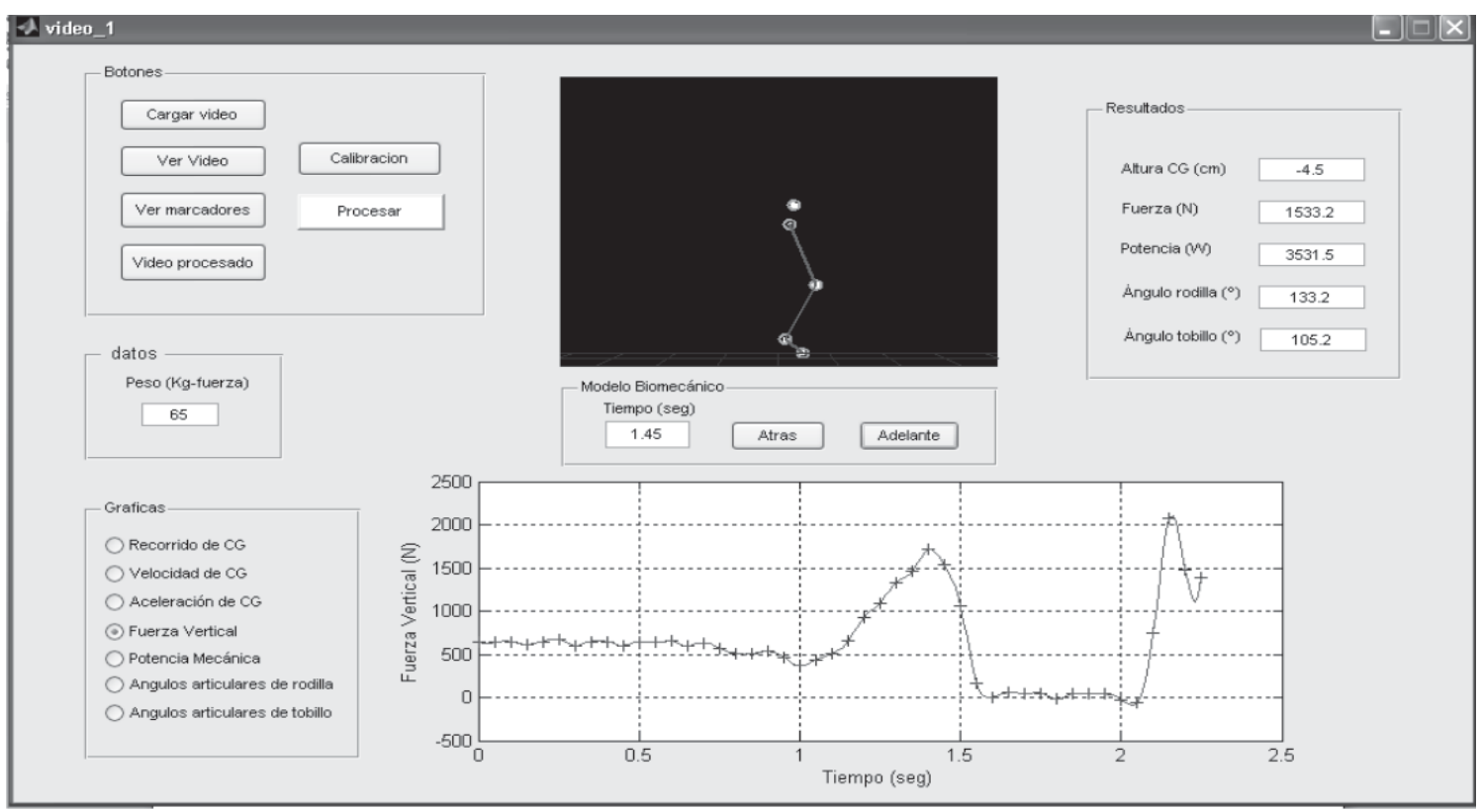

Figura 7. Panel central del software 
A continuación se muestran los resultados de un deportista que pesó $65 \mathrm{Kg}$, el cual realizó un salto con contra movimiento. En la Figura 8 se observa el video procesado, y en las Figuras 9, 10, 11, 12 y 13 se observan las variables obtenidas.

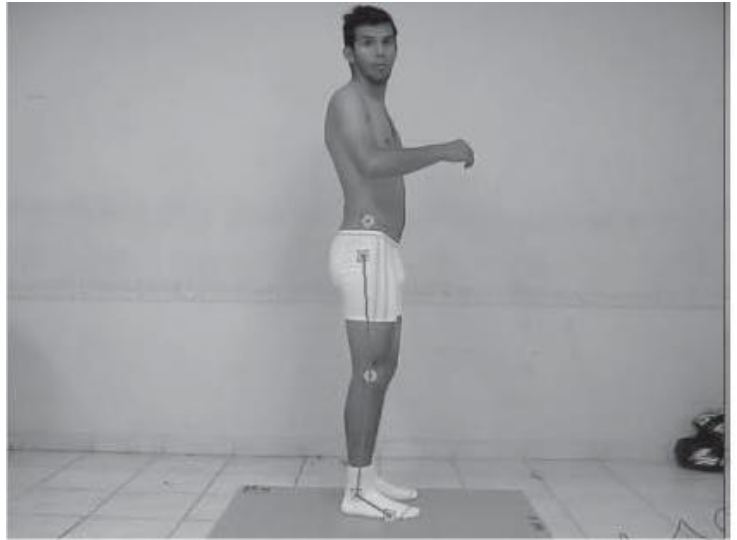

Figura 8. Video procesado

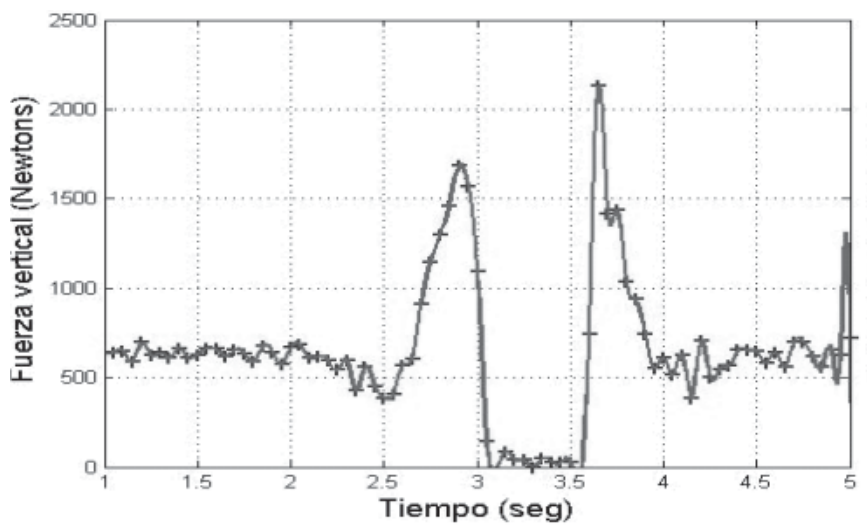

Figura 10. Fuerza vertical

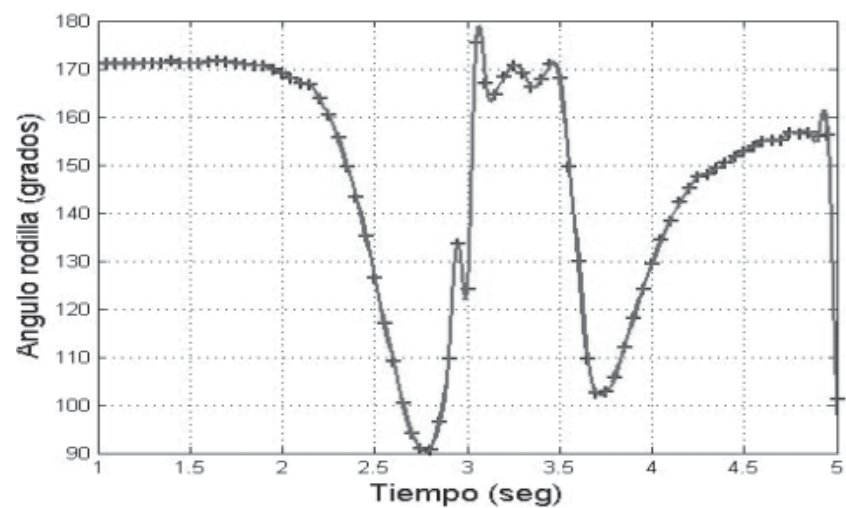

Figura 12. Ángulo articulación rodilla

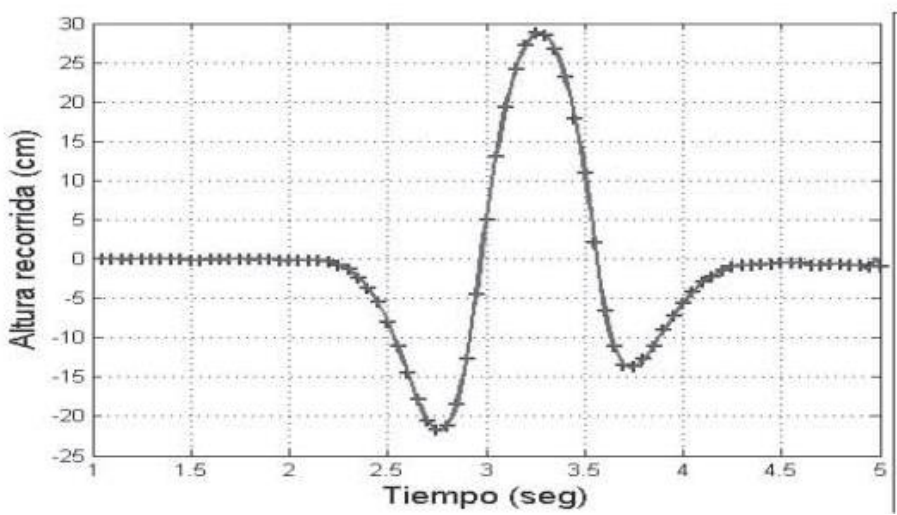

Figura 9. Altura recorrida CG

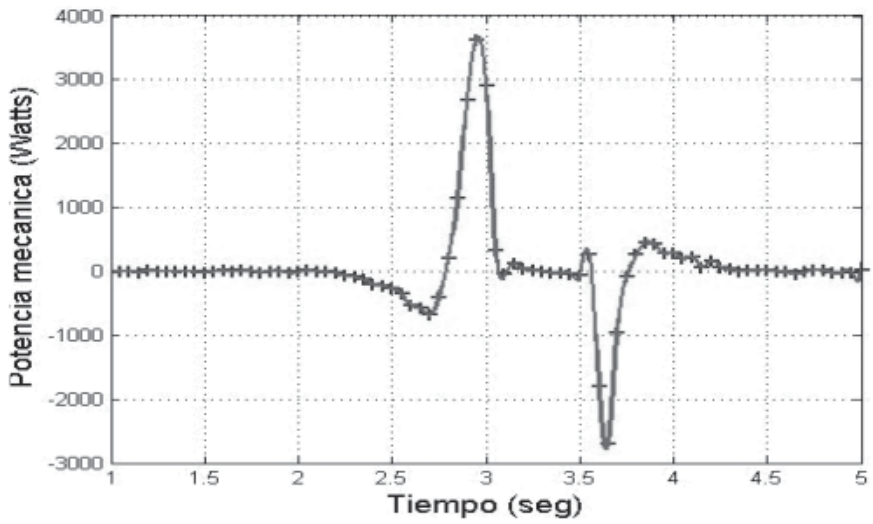

Figura 11. Potencia mecánica

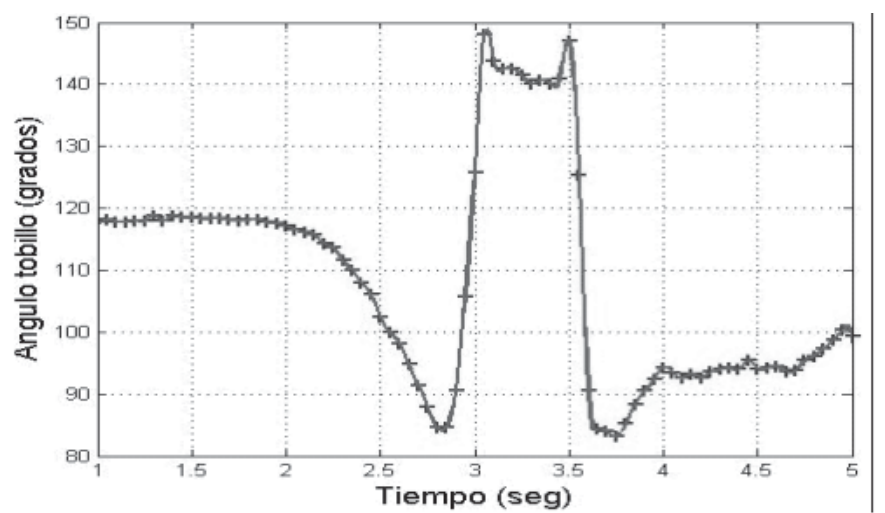

Figura 13. Ángulo articulación tobillo

\subsection{Validación del Sistema}

Con el fin de comprobar que los datos que se obtienen del sistema de videogrametría son equivalentes con datos Obtenidos en sistemas comerciales, se realizaron tres tipos de pruebas las cuales se enunciaran a continuación. 
3.3.1 Variables Cinemáticas. Las variables cinemáticas se validaron con el sistema de evaluación cinemática AxonJump que consta de una alfombra sobre la cual el sujeto salta y por medio del software se visualiza Tiempo de vuelo en $\mathrm{ms}$, Altura alcanzada en $\mathrm{cm}$ y Velocidad de despegue en $\mathrm{m} / \mathrm{s}$. El procedimiento para la validación fue poner a saltar a cinco sujetos sobre la plataforma AxonJump siguiendo el protocolo de filmación. Obtenidos los resultados se prosiguió a comparar los datos de altura alcanzada y velocidad de despegue con los suministrados por el sistema de videogrametría. En la tabla 1 se calcula el porcentaje de error de la altura máxima y la velocidad de despegue del sistema de videogrametría con respecto al sistema AxonJump. Se expresa con un error medio y su respectiva desviación estándar. En ambos casos se observa que los errores están dentro del rango medio según la literatura.

Tabla 1. Porcentaje de error de altura y velocidad del Sistema de videogrametría

\begin{tabular}{|c|c|c|}
\hline & ERROR MEDIO $(\boldsymbol{\%})$ & DESV. EST (\%) \\
\hline ALTURA $(\mathrm{cm})$ & 4.27 & \pm 2.39 \\
\hline VELOCIDAD $(\mathrm{m} / \mathrm{s})$ & 9.20 & \pm 5.22 \\
\hline
\end{tabular}

La variable cinemática de la aceleración se valida de forma empírica, debido a que en el momento en que los sujetos empiezan a caer en el tiempo de vuelo, los valores tomados en éste momento son una constante que reflejan la gravedad terrestre (aproximadamente $9.8 \mathrm{~m} / \mathrm{s}^{2}$ ), por lo tanto se asume que los datos son correctos.

3.3.2 Variables Cinéticas. Debido a que en el laboratorio ALTIUS no se disponía de plataformas de fuerzas, se recurrió a validar esta variable de forma gráfica con otras obtenidas en prácticas de biomecánica realizadas en la Universidad de Castilla de la Mancha en el cual utilizan una plataforma de fuerzas portable QuattroJump de Kistler (Aguado y Alegre, 2000).En la Figura 14 se observan cuatro características muy importantes en el desarrollo del salto y que se repite en la Figura 10 generada por el sistema de videogrametría:

- El Peso del sujeto: se obtiene siempre que el sujeto se encuentre en reposo, debido a que es la fuerza que ejerce la gravedad sobre su masa corporal.

- El Pico 1: es la Fuerza en el punto más bajo del contra movimiento (manifestación activa), en esta fase el sujeto ejerce el mínimo ángulo articular en la rodilla.

- La Fase de vuelo: es el tiempo en el cual el sujeto no ejerce ninguna fuerza, debido a que su cuerpo no está en contacto con el suelo.

- El Pico 2: es la Fuerza máxima ejercida cuando el sujeto entra en contacto con el suelo (manifestación reactiva) luego de la fase de vuelo.

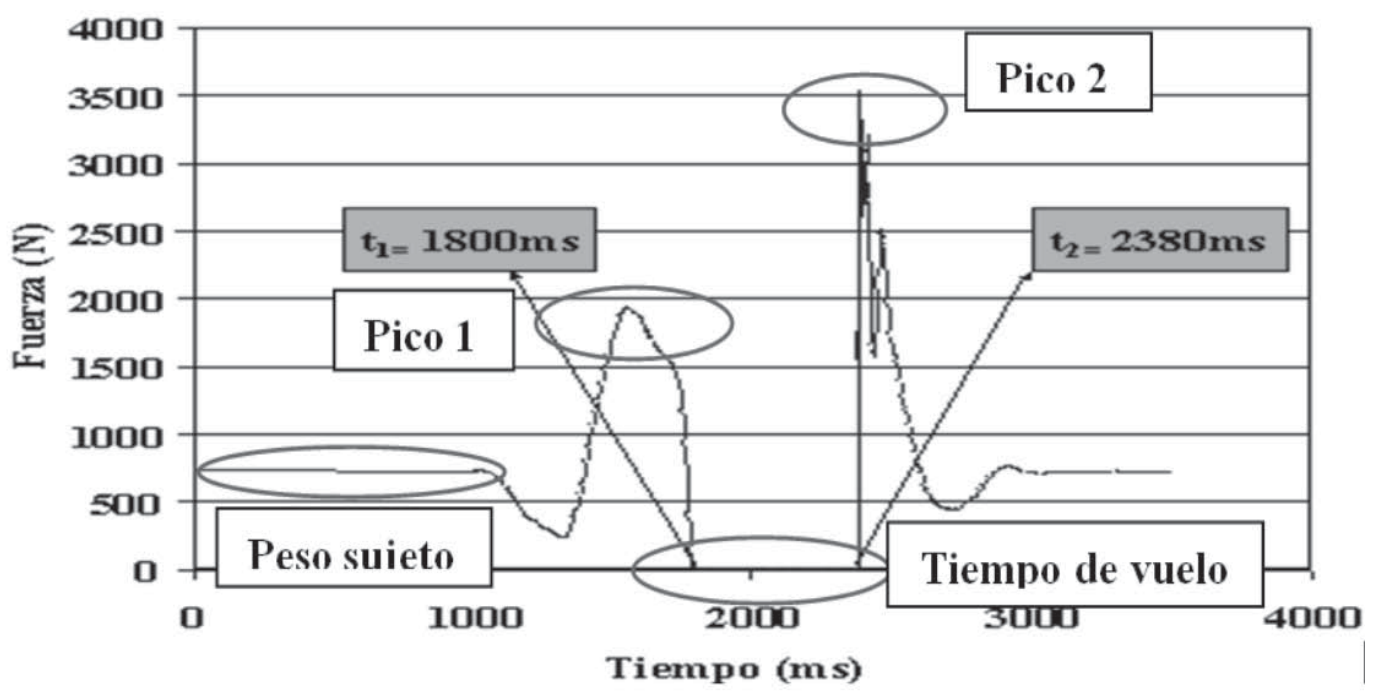

Figura 14. Fuerza vertical obtenida de plataforma de fuerza Fuente: García y Peleteiro 2004

El valor de la potencia máxima ejercida en un salto vertical se puede comparar con el valor de la Ecuación de Lara (Aguado y Alegre, 2000)para calcular la potencia mecánica. En la tabla 2 se calcula el porcentaje de error de la 
Potencia mecánica del sistema de videogrametría con respecto a la ecuación de Lara. Se expresa con un error medio y su respectiva desviación estándar. Se observa que el error está dentro del rango medio según la literatura.

Tabla 2. Porcentaje de error potencia mecánica del sistema de videogrametría

\begin{tabular}{|c|c|c|}
\hline & ERROR MEDIO (\%) & DESV. EST (\%) \\
\hline POTENCIA MECÁNICA $(\mathrm{W})$ & 5.60 & \pm 3.46 \\
\hline
\end{tabular}

3.3.3 Ángulos Articulares. La validación de los ángulos articulares de rodilla y tobillo se realizó utilizando un goniómetro de brazos. El procedimiento que se siguió fue medir el ángulo que se formaban entre los marcadores adyacentes a la articulación. Así para el ángulo articular de la rodilla se referenció el segundo marcador (TM) y el cuarto marcador (ML) manteniendo el centro del instrumento en el tercer marcador (EFL); y para medir el ángulo articular del tobillo se referenció el tercer marcador (EFL) y el quinto marcador (MET), manteniendo el centro del instrumento en el cuarto marcador (ML). En la tabla 3 se muestra el porcentaje de error de los ángulos articulares de posición inicial y de despegue del sistema de videogrametría con respecto a las mediciones del goniómetro. Se expresa con un error medio y su respectiva desviación estándar.En ambos casos se observa que los errores están dentro del rango medio según la literatura.

Tabla 3. Porcentaje de error de ángulos articulares de posición inicial y de despegue del sistema de videogrametría con referencia a las mediciones del goniómetro

\begin{tabular}{|c|c|c|}
\hline & ERROR MEDIO (\%) & DESV. EST (\%) \\
\hline ÁNGULO RODILLA $\left({ }^{\circ}\right)$ & 3.09 & \pm 1.08 \\
\hline ÁNGULO TOBILLO $\left({ }^{\circ}\right)$ & 7.20 & \pm 6.33 \\
\hline
\end{tabular}

\section{Conclusiones}

Se logró diseñar e implementar un sistema de videogrametría digital bidimensional que permite visualizar la medición de la altura máxima alcanzada por un deportista y a partir de esta variable obtener características cinemáticas como: velocidad de despegue y aceleración del centro de gravedad; cinéticas como: fuerza vertical y potencia mecánica; y por último ángulos articulares de rodilla y tobillo en la ejecución del salto vertical.

Se generó un protocolo de filmación completo para la prueba de salto, este incluye ubicación de marcadores corporales, dimensiones del escenario y procedimiento propio para ejercer tres clases de saltos que no ocultan ningún marcador corporal.

Con la implementación de este sistema a muy bajo costo, el cual solo requiere de una cámara y un software de procesamiento, se pretende integrar y mejorar la velocidad al momento de recolectar datos, debido a que este sistema obtiene las variables más importantes para evaluar un salto vertical, ya que en la actualidad se requieren diferentes instrumentos para integrar todas las variables a analizar, tal es el caso de la plataforma de vuelo para medición de alturas, plataformas de fuerzas para medición de fuerza y potencia musculares, e instrumentos como goniómetros para medición de ángulos articulares.

Con la evaluación de las gráficas y animación en dos dimensiones mostradas por el software, los entrenadores de deportistas pueden empezar a realizar sus recomendaciones de acuerdo a las falencias observadas en la ejecución del salto vertical, con el fin de mejorar la potencia mecánica de acuerdo a la clase de deporte realizado, por ejemplo voleibolistas o futbolistas de alto rendimiento de la región.

\section{Referencias Bibliográficas}

1. Acevedo, D.; Hincapié, F.; Sánchez, J., 2008.Valoración de la manifestación reactiva de la fuerza de los miembros inferiores a las integrantes de la selección Antioquia de voleibol categoría junior rama femenina. Universidad de Antioquia. Instituto de educación física. Medellín.

2. Aguado, Xavier., Alegre, Luis, 2000. Potencia máxima en salto (medida vs. calculada). Cuaderno de prácticas de Biomecánica de las Técnicas deportivas. Facultad de ciencias del deporte. Universidad de Castilla de la Mancha. 
3. Calderón, Ximena y Montero, María., 2004. Correlación entre torque isocinético máximo de cuádriceps y Potencia de la cadena muscular de la extremidad inferior. Facultad de medicina. Universidad de chile.

4. Díaz C., Torres A. ,Ramírez J., García L., Álvarez N., 2006. Descripción de un dispositivo destinado al análisis de la marcha en dos dimensiones, Escuela de Ingeniería de Antioquia, Medellín (Colombia). Revista EIA, ISSN 1794-1237 Número 5 p. 85-92.

5. García López, J.; Peleteiro J., 2004. Test de salto vertical (II):Aspectos biomecánicos. Rendimiento Deportivo. Com, $\mathrm{N}^{\circ} 7$.

6. Garzón, Carolina., Cruz, Fabiola.,2005.Diseño e implementación de un sistema electrónico para la medición de las presiones plantares. Universidad Surcolombiana. Ingeniería Electrónica. Neiva.

7.Martínez C. Fabio., 2009. Modelo de Seguimiento del Centro de Masa para el Análisis Cinemático de la Marcha. Trabajo de grado Maestría en Ingeniería Biomédica. Universidad Nacional de Colombia. Bogotá.

8.Ortiz, J. Carlos., 2004.Influencia del calentamiento previo sobre la saltabilidad en tenistas universitarios. Facultad de Ciencias de la Rehabilitación. Universidad Andrés Bello, Viña del Mar, Chile.

9.Rodríguez, C.; Quintero, Hugo.; Gutiérrez, D; Ashner, H., 2005. Desarrollo de una herramienta de análisis de movimiento para brazo humano. Departamento de ingeniería mecánica, universidad de los andes (Colombia). biomecánica, 13 (1), pp. 51-57.

10. Sánchez, A; Aguilar Martín, J; Martínez Latorre, M; Fernández Sora, A; Cajal Hernando, C., 2005. Desarrollo de un procedimiento de caracterización de sistemas de análisis del movimiento humano. Universidad de Zaragoza, España. Departamento de ingeniería de diseño y fabricación. 